\title{
How Things Happen
}

\author{
Martin Fried ${ }^{\mathrm{a}}$ Volkan Yumuk ${ }^{\mathrm{b}}$ \\ ${ }^{a}$ OB Klinika, Centre for Treatment of Obesity and Metabolic Disorders, 1st Faculty \\ of Medicine, Charles University, Prague, Czech Republic, ${ }^{b}$ Division of Endocrinology, \\ Metabolism and Diabetes, Istanbul University Cerrahpasa Medical Faculty, Istanbul, Turkey
}

In the year 2008 I was honoured and delighted to be invited to write an editorial in OBESITY FACTS on the occasion of International Federation for the Surgery of Obesity and Metabolic Disorders - European Chapter (IFSO-EC) members joining the Editorial Board of OBESITY FACTS, and therefore the journal becoming an affiliated journal of the IFSO-EC. The 2008 editorial was entitled 'The Circle Is Closing - OBESITY FACTS Launched as Official Journal of IFSO-EC'. The editorial pointed out some milestones which finally enabled the European bariatric surgical community to accomplish acceptance into the large, non-surgical, medical 'obesitology family' after a more than half a century long struggle. By that time, my feeling was that the most important goals in the field of interdisciplinary cooperation were already achieved, so we may enjoy the fruits of it.

However, 5 years later, I am once again and even more proud, pleased and honoured to be given the opportunity to jointly write, together with Professor Yumuk, a second editorial in OBESITY FACTS. Probably the best title for today's editorial may have been something like 'The Circle Is Expanding Exponentially'. My dear friend Volkan and I will try to explain you why.

Medico-Surgical cooperation, under the umbrella of Europe's two largest scientific societies, the European Society for the Study of Obesity (EASO) and the IFSO-EC, especially in the field of obesity research and management has accelerated with a speed that was almost impossible to imagine. Let's have a quick look at the history. The IFSO-EC was founded in the year 2004 at the 13th European Congress on Obesity that was held in Prague. Our friends from EASO were kindly hosting at their official meeting a small group of bariatric surgeons who had the vision of forming the IFSO-EC.

One year later, a group of experts constituted by the two European societies (EASO and IFSO-EC) wrote the 'Interdisciplinary European Guidelines for Surgery Of Severe Obesity', which were published in several peer-reviewed journals between the years 2007 and 2008. An intensive exchange of views and ideas was started between the two societies. A few tens of key opinion leaders from both societies took part in the respective societies' meetings, gave talks, shared experience on the oucomes reached by medical and surgical treatment modalities of obesity.

Later on, in 2011 in Munich, Germany, and in 2013 in Liverpool, UK, EASO and IFSO-EC joint Medico-Surgical Workshops were organised with great success. The workshops estab- 
lished a platform for truly interdisciplinary debates and open exchange of ideas. It was decided that the workshops will be held annually under the alternative leadership of each society. As a result of the 2013 workshop, new, up-dated and revised 'Interdisciplinary European Guidelines on Metabolic and Bariatric Surgery' were created by an expert panel of EASO and IFSO-EC members. The guidelines are presented in this issue of OBESITY FACTS to all readers.

Type 2 diabetes mellitus is one of the most prominent fields where the medico-surgical treatment efforts are gradually melting in a pot which is still hot. Several decades ago the U.K. Prospective Diabetes Study (UKPDS) was mainly designed to determine whether improved blood glucose control in type 2 diabetes will prevent diabetes complications. It really worked out well for microvascular complications. However, a UKPDS overview of 6 years' therapy of type 2 diabetes reported that progressively increasing hyperglycaemia, which is associated with decreasing beta cell function, was a marked feature irrespective of the therapy used (conventional therapy-diet only vs. intensive medical therapy). Thus, with these findings the clinicians came to the conclusion that type 2 diabetes is a progressive disease, and the researchers have plunged into the pursuit of discovering novel therapies either halting or reversing this progression ever since [1]. Recently a metabolic sub-study of the Surgical Therapy and Medications Potentially Eradicate Diabetes Efficiently (STAMPEDE) trial has compared bariatric surgery with intensive medical therapy in obese patients with type 2 diabetes. The 2-year results demonstrated a significant improvement in beta cell function in patients followed up in the surgical arm of the study [2]. We find the journey from the UKPDS to the STAMPEDE trial quite fascinating but are cautiously waiting for more, robust evidence from future randomised clinical trials (RCTs).

Diabetes remission actually means resolution of glycaemic parameters. To put it another way it is reaching the targets and staying there so that complications are avoided or they regress. It seems that talking about a cure is too early. Post-bariatric surgery diabetes remission rates were considerably high in previous studies. There were inter-study variations regarding the definition for remission. Recent RCTs have adopted the ADA Consensus criteria for diabetes remission, hence bringing in a standard end point [3].

Although the outcome studies concerning the diabetic complications after metabolic surgery are scarce, an un-blinded, case-controlled trial with 10-year follow up demonstrated that renal and cardiovascular complications were dramatically reduced in the surgical arm, indicating long-term benefits of bilio-pancreatic diversion on diabetic complications in the case of morbid obesity with decompensated diabetes [4]. The review of the key results from the Swedish Obese Subjects (SOS) trial showed that, compared to conventional therapy, bariatric surgery was associated with a long-term reduction in overall mortality and decreased incidence of diabetes, myocardial infarction, stroke and cancer [5].

EASO and IFSO-EC are convinced that the only way forward is based on the paradigm that physicians and surgeons must work in collaboration and follow the joint guidelines produced based on current scientific evidence in cooperation of IFSO-EC and EASO whose members have been successfully working together in accordance with a perfect physician-surgeon team spirit.

\section{References}

$\checkmark 1$ U.K. Prospective Study Group: Overview of 6 years' therapy of type 2 diabetes: a progressive disease. Diabetes 1995;44:1249-1258.

-2 Kashyap SR, Bhatt DL, Wolski K, et al: Metabolic effects of bariatric surgery in patients with moderate obesity and type 2 diabetes. Diabetes Care 2013;36:2175-2182.

3 Buse JB, Caprio S, Cefalu WT, et al: How do we define cure of diabetes? Diabetes Care 2009;32:2133-2135.

4 Iaconelli A, Panunzi S, De Gaetano A, et al: Effects of biliopancreatic diversion on diabetic complications: a 10 year follow-up. Diabetes Care 2011;34:561-567.

5 Sjöström LJ: Review of the key results from the Swedish Obese Subjects (SOS) trial - a prospective controlled intervention study of bariatric surgery. Intern Med 2013;273:219-234. 